\title{
Joule Inversion Temperatures for Some Simple Real Gases
}

\author{
E. Albarrán-Zavala ${ }^{1, *}$, B. A. Espinoza-Elizarraraz ${ }^{2}$ and F. Angulo-Brown ${ }^{1}$ \\ ${ }^{I}$ Departamento de Física, Escuela Superior de Física y Matemáticas, ESFM-IPN \\ ${ }^{2}$ Departamento de Ingeniería de Sistemas, Escuela Superior de Ingeniería Mecánica y Eléctrica, ESIME-IPN. Instituto \\ Politécnico Nacional, U. P. Adolfo López Mateos, C. P. 07738, México D. F.
}

\begin{abstract}
In the present work we calculate inversion temperatures $T_{i}$, for some simple real gases $\left(\mathrm{He}, \mathrm{Ne}, \mathrm{Ar}, \mathrm{Kr}\right.$ and $\left.\mathrm{H}_{2}\right)$ in the case of a Joule expansion; that is, a free adiabatic expansion. These calculations are made by means of an intermolecular potential of the Lennard-Jones type $(12,6)$, slightly modified by using a Jagla linear ramp in the repulsive part of the potential. For the Helium we find a $T_{i}$ in agreement with both previous calculations published by other authors and with experimental results. For $\mathrm{Ne}$ and Ar our results are also within the interval of values previously reported, and for $\mathrm{Kr}$ we also obtain a very high inversion temperature as some other authors. However, for $\mathrm{H}_{2}$ we find a $T_{i}$ approximately twice to big than its estimated experimental value. This last calculation is corrected if we use a short ranged Lennard-Jones potential of the type $(24,12)$, obtaining a result in good agreement with the recognized experimental value.
\end{abstract}

\section{INTRODUCTION}

It is well known that all real gases undergoing a throttling process (the so-called Joule-Kelvin expansion) have an inversion temperature, $T_{i}$; that is, a temperature at which the process changes from a heating to a cooling process. This temperature depends upon the particular gas and upon both the initial and final pressures [1]. On the other hand, only in some few references [2-4], the problem of an inversion temperature associated to the so-called Joule expansion (free adiabatic expansion) for real gases is discussed. In fact, Goussard and Roulet [2] asserted that there exists an inversion temperature, $T_{i}$, for all real gases undergoing an adiabatic free expansion. If $T_{0}$ is the initial temperature, then for $T_{0}<T_{i}$, the gas is cooler after a free expansion and for $T_{0}>T_{i}$, the gas is warmer. According to Goussard and Roulet [2], all real gases which can be described by intermolecular potentials $u(r)$ which diverge with small values for $r$ at least like $r^{-3}$, necessarily have an inversion temperature. For example, for the Lennard-Jones model $u(r) \sim r^{-12}$ for $r$ $\rightarrow 0$, and they used this potential to calculate $T_{i}$ for helium and hydrogen [2]. Their result for the helium case is excellent according to experimental variations of the second virial coefficient $B(T)$ measured by Holborn and Otto [5] and also given in [1]. However, for the hydrogen case they calculated $T_{i}$ twice to big than its estimated experimental value [1, 5]. Boschi-Filho and Buthers [3] studied the Joule inversion temperature for several simple real gases at high temperatures based on the $B(T)$ behavior and they suggested that most of simple real gases have an inversion temperature corresponding to the maximum value of $B(T)$ in a range of temperatures so high that few experimental data are available. Recently, Parsafar, et al. [4] proposed a method to

*Address correspondence to this author at the Departamento de Física, Escuela Superior de Física y Matemáticas, ESFM-IPN. Instituto Politécnico Nacional, U. P. Adolfo López Mateos, C. P. 07738, México D. F.; Tel: +52 (55) 57296000 ext 55034; E-mail: caesar_erik@yahoo.com modify hard-core potentials to predict real fluid properties at low densities, including the inversion temperature. In the present article we discuss the problem of the Joule inversion temperature by means of a Lennard-Jones potential slightly modified in its repulsive and positive part according to the so-called Jagla potential [6]. This potential allows to obtain analytical expressions for the second virial coefficient and for its first derivative $d B / d T$, necessary to calculate the Joule inversion temperature. With this potential we calculate $T_{i}$ for $\mathrm{He}, \mathrm{Ne}, \mathrm{Ar}, \mathrm{Kr}$ and $\mathrm{H}_{2}$.

Our results for $\mathrm{He}, \mathrm{Ne}$ and $\mathrm{Ar}$ are in agreement with [2] and [3] respectively, and for Kr we obtain a very high inversion temperature as in [3]. For the hydrogen case we also obtain a $T_{i}$ value twice to big the recognized experimental value reported in [1]. However, we recuperate a correct result by using a Lennard-Jones potential with exponents $(24,12)$. The article is organized as follows: In section 2 we present the Lennard-Jones modified model for the intermolecular potential. In section 3 we use experimental data to fit our approximated model to calculate $T_{i}$. In section 4 , we calculate $T_{i}$ for hydrogen by using a $(24,12)$ Lennard-Jones potential. Finally we present our concluding remarks.

\section{THE MODIFIED LENNARD-JONES POTENTIAL}

The temperature change for a gas undergoing an adiabatic free expansion can be calculated by means of the so-called Joule coefficient,

$$
J=\left(\frac{\partial T}{\partial V}\right)_{E}=-\left(\frac{\partial T}{\partial E}\right)_{V}\left(\frac{\partial E}{\partial V}\right)_{T}=-\frac{1}{C_{V}}\left(\frac{\partial E}{\partial V}\right)_{T}
$$

being $T$ the absolute temperature, $V$ the volume, $E$ the internal energy and $C_{V}$ the heat capacity at constant volume. Since $C_{V}$ is always positive, the sign of the temperature variation along the expansion is determined by the sign of $(\partial E / \partial V)_{T}$. The coefficient $J$ can also be calculated knowing $C_{V}$ and the gas state equation [2], 
$J=\frac{1}{C_{V}}\left[P-T\left(\frac{\partial P}{\partial T}\right)_{V}\right]$

with $P$ the gas pressure. Writing the virial expansion as

$P V=n R T\left(1+\frac{n B}{V}+\frac{n^{2} C}{V^{2}}+\frac{n^{3} D}{V^{3}}+\cdots\right)$

which expresses the deviation of $P$ from that of an ideal classical gas, it is easy to show, that in the thermodynamic limit $V \rightarrow \infty$ [3], the Joule coefficient can be expressed as

$J=-\frac{n^{2} R T^{2}}{C_{V} V^{2}}\left(\frac{d B}{d T}\right)$

being $B=B(T)$ the second virial coefficient and $n$ the molar number. If an inversion temperature $T_{i}$ exists then,

$\left(\frac{d B}{d T}\right)_{T_{i}}=0$

Boschi-Filho and Buthers [3] showed that some state equations such as Van der Waals, Berthelot, Dieterici, Redlich-Kwong and Beattie-Bridgeman have not an inversion temperature. This fact according to Goussard and Roulet [2] occurs because for real gases the existence of an inversion temperature comes directly from the fact that there is a high but not infinite repulsive part in $u(r)$. On the other hand, for a hard sphere repulsive part, $u(r)=\infty$ for $r<\sigma$ and therefore $(\partial E / \partial V)_{T}$ is always positive in their Eq. (6), [2]. This is clearly the case for a Van der Waals gas, which always cools in a Joule expansion [2].

As is well known [7], the second virial coefficient can be written as

$$
B=2 \pi N_{A} \int_{0}^{\infty}\left(1-e^{-u / k T}\right) r^{2} d r
$$

where $u(r)$ is the intermolecular potential, $k$ the Boltzmann's constant, $N_{A}$ the Avogadro's number, and $r$ is the distance between a pair of molecules. In the present article we use the Lennard-Jones $(\mathrm{LJ})$ potential, that is,

$$
u(r)=4 \varepsilon\left[\left(\frac{\sigma}{r}\right)^{12}-\left(\frac{\sigma}{r}\right)^{6}\right]
$$

being $\varepsilon$ the well depth and $\sigma$ the point where $u(r)=0$. We slightly modify the LJ-potential following the procedure of Jagla [6], which is depicted in Fig. (1), and is given by:

$$
u(r)=\left\{\begin{array}{ll}
+\infty & \text { if } \quad 0 \leq r \leq \lambda \\
\alpha(r-\sigma) & \text { if } \quad \lambda \leq r \leq \sigma \\
4 \varepsilon\left[\left(\frac{\sigma}{r}\right)^{12}-\left(\frac{\sigma}{r}\right)^{6}\right] & \text { if } \quad \sigma \leq r \leq+\infty
\end{array}\right\}
$$

$\alpha=\left(\frac{4 \varepsilon}{\lambda-\sigma}\right)\left[\left(\frac{\sigma}{\lambda}\right)^{12}-\left(\frac{\sigma}{\lambda}\right)^{6}\right]$

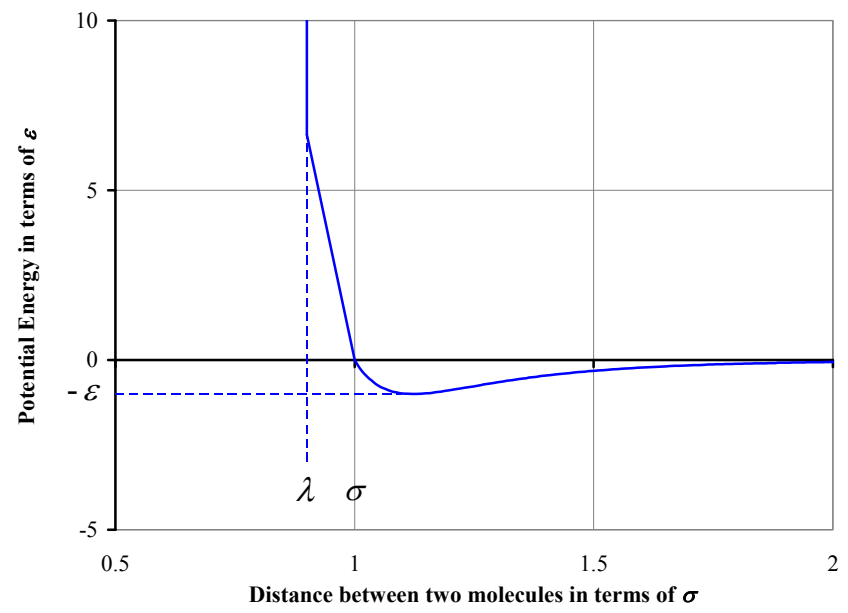

Fig. (1). The modified LJ-potential. The function is composed by three parts. The first part is in the interval corresponding to $0<r<$ $\lambda$ where the function is $+\infty$. The second part is formed by a ramp with negative slope for $\lambda<r<\sigma$. Finally, the third part is the LJpotential in the interval corresponding to $\sigma<r<+\infty$.

For this potential we propose the following approximations: for the interval $0<r<\lambda$, the term $1-e^{-\beta u}$ inside the integral of Eq. (6) can be approximated by

$1-e^{-\beta u} \approx 1$

being $\beta=1 / k T$. For the interval $\lambda<r<\sigma$ the term $1-e^{-\beta u}$ is maintained as it is. And finally, in the interval $\sigma<r<+\infty$, for the case $|\beta u| \ll<1$, we take:

$1-e^{-\beta u} \approx \beta u$

By using these approximations, Eq. (6) can be written as,

$B(T)=2 \pi N_{A} \int_{0}^{\lambda} r^{2} d r+2 \pi N_{A} \int_{\lambda}^{\sigma}\left(1-e^{-\beta u}\right) r^{2} d r$

$+2 \pi N_{A} \int_{\sigma}^{+\infty} \beta u r^{2} d r$

which leads to

$$
\begin{aligned}
& B=\frac{2}{3} \pi N_{A} \sigma^{3}-\frac{16 \pi \varepsilon N_{A} \sigma^{3}}{9 k T}+\frac{2 \pi N_{A} k T}{\alpha}\left[\sigma^{2}-\lambda^{2} e^{\alpha \beta(\sigma-\lambda)}\right] \\
& +\frac{4 \pi N_{A} k^{2} T^{2}}{\alpha^{2}}\left[\sigma-\lambda e^{\alpha \beta(\sigma-\lambda)}\right]+\frac{4 \pi N_{A} k^{3} T^{3}}{\alpha^{3}}\left[1-e^{\alpha \beta(\sigma-\lambda)}\right]
\end{aligned}
$$

The first derivative of $B(T)$ is immediately obtained,

$$
\begin{aligned}
& \frac{d B}{d T}=\frac{16 \pi \varepsilon N_{A} \sigma^{3}}{9 k T^{2}}+\frac{2 \pi N_{A} k}{\alpha}\left[\sigma^{2}-\lambda^{2} e^{\alpha \beta(\sigma-\lambda)}\right] \\
& +\frac{8 \pi N_{A} k^{2} T}{\alpha^{2}}\left[\sigma-\lambda e^{\alpha \beta(\sigma-\lambda)}\right]+\frac{12 \pi N_{A} k^{3} T^{2}}{\alpha^{3}}\left[1-e^{\alpha \beta(\sigma-\lambda)}\right] \\
& +\frac{2 \pi N_{A} k T}{\alpha}\left[\frac{\alpha(\sigma-\lambda) \lambda^{2} e^{\alpha \beta(\sigma-\lambda)}}{k T^{2}}\right] \\
& +\frac{4 \pi N_{A} k^{2} T^{2}}{\alpha^{2}}\left[\frac{\alpha(\sigma-\lambda) \lambda e^{\alpha \beta(\sigma-\lambda)}}{k T^{2}}\right]
\end{aligned}
$$


$+\frac{4 \pi N_{A} k^{3} T^{3}}{\alpha^{3}}\left[\frac{\alpha(\sigma-\lambda) e^{\alpha \beta(\sigma-\lambda)}}{k T^{2}}\right]$

In the following section we shall use these algebraic results to obtain the Joule inversion temperatures for several simple real gases. We will plot $B(T)$ and $f(T)=(T$ $\left.{ }^{2} / 2 \pi\right) \times(d B / d T)$ against $T$ for several gases (He, Ne, $\mathrm{Ar}, \mathrm{Kr}$ and $\mathrm{H}_{2}$ ).

\section{JOULE INVERSION TEMPERATURES}

In this section we shall use Eqs. (13) and (14) to calculate the Joule inversion temperature for several simple real gases ( $\mathrm{He}, \mathrm{Ne}, \mathrm{Ar}, \mathrm{Kr}$, and $\mathrm{H}_{2}$ ).

\section{A) Helium}

For this gas we take experimental data for $B(T)$ reported by White et al. [8] for obtaining $\lambda, \sigma$ and $\varepsilon$ in Eq. (13) as fitting parameters. In Fig. (2) we see the experimental points of $B(T)$ and the fitted curve given by Eq. (13). The resulting values of the parameters are $\lambda=2.002807 \times 10^{-10} \mathrm{~m}, \sigma=$ $2.477172 \times 10^{-10} \mathrm{~m}$, and $\varepsilon=1.260382 \times 10^{-22} \mathrm{~J}$, which means $\varepsilon / k=9.1289 \mathrm{~K}$. From quantum mechanics calculations, Poling et al. [9] give for the helium $\varepsilon / k=10.22 \mathrm{~K}$. In Fig. (3) we depict $f(T)=\left(T^{2} / 2 \pi\right) \times(d B / d T)$ against $T$ according to Eq. (14). The inversion temperature is given by the point where $d B / d T=0$ and it corresponds to $T_{i} \approx 177 \mathrm{~K}$. Goussard and Roulet [2] for a LJ-potential, obtained an inversion temperature given by $T_{i} \approx 25 \varepsilon / k$, which for He gives $T_{i} \approx 200 \mathrm{~K}$ (See Figs. 2 and 3).

\section{B) Neon}

In this case we took $B(T)$ experimental data from Prausnitz et al. [7] and Dymond and Smith [10]. In Fig. (4), we depict the fitted curve, Eq. (13), to experimental data obtaining the following values for the parameters $\lambda=$ $2.027787 \times 10^{-10} \mathrm{~m}, \quad \sigma=2.571497 \times 10^{-10} \mathrm{~m}$, and $\varepsilon=$ $6.311761 \times 10^{-22} \mathrm{~J}$, which give $\varepsilon / k=45.7158 \mathrm{~K}$. From viscosity data, Poling et al. [9] report for Neon $\varepsilon / k=32.8 \mathrm{~K}$. In Fig. (5) we obtain an inversion temperature of $T_{i} \approx 867 \mathrm{~K}$, which is within the $T_{i}$-interval given by ref. [3], for Neon $870 \pm 390$ $\mathrm{K}$.

\section{C) Argon}

In this case we take experimental second virial data given by Fender and Halsey [11] (see Fig. 6). The resulting fitted parameters according with Eq. (13) are $\lambda=3.145084 \times 10^{-10}$ $\mathrm{m}, \sigma=4.74678 \times 10^{-10} \mathrm{~m}$, and $\varepsilon=1.231451 \times 10^{-21} \mathrm{~J}$, which give $\varepsilon / k=89.1935 \mathrm{~K}$. From viscosity data, Poling et al. [9] find $\varepsilon / k=93.3 \mathrm{~K}$. In Fig. (7) we have $f(T)$ versus $T$, and we obtain $T_{i} \approx 3448 \mathrm{~K}$ which is within the $T_{i}$-interval reported by Boschi-Filho and Buthers [3] for Argon, $T_{i} \approx 3800 \pm 1800$ K.

\section{D) Krypton}

For this gas we use $B(T)$ experimental data also given by Fender and Halsey [11] (see Fig. 8). The resulting fitted parameters by using Eq. (13) are $\lambda=3.631624 \times 10^{-10} \mathrm{~m}$, $\sigma=6.153939 \times 10^{-10} \mathrm{~m}$, and $\varepsilon=1.276291 \times 10^{-21} \mathrm{~J}$, which give $\varepsilon / k=92.4413 \mathrm{~K}$. From viscosity data, Poling et al. [9] obtain $\varepsilon / k=178.9 \mathrm{~K}$. In Fig. (9) we see that $T_{i}$ for krypton seemingly has a very high value, $T_{i} \approx 6481 \mathrm{~K}$. Boschi-Filho and Buthers [3] assert that at least for the temperature range $0-4000 \mathrm{~K}$ this gas does not show a maximum in its second virial coefficient. However, for $T_{i}>4000 \mathrm{~K}$ we find a possible inversion temperature.

\section{E) Hydrogen}

In the hydrogen case we found an inversion temperature by taking $B(T)$ experimental data from Prausnitz et al. [7] and Dymond and Smith [10]. By means of Eqs. (13) and (14) we obtained $T_{i} \approx 922 \mathrm{~K}$. The fitted parameters are $\lambda=$ $2.368190 \times 10^{-10} \mathrm{~m}, \sigma=2.861934 \times 10^{-10} \mathrm{~m}$, and $\varepsilon=$ $5.513926 \times 10^{-22} \mathrm{~J}$, which give $\varepsilon / k=39.9372 \mathrm{~K}$. On the other hand, from viscosity data, Poling et al. [9] report $\varepsilon / k=59.7$ $\mathrm{K}$ (See Figs. 10 and 11).

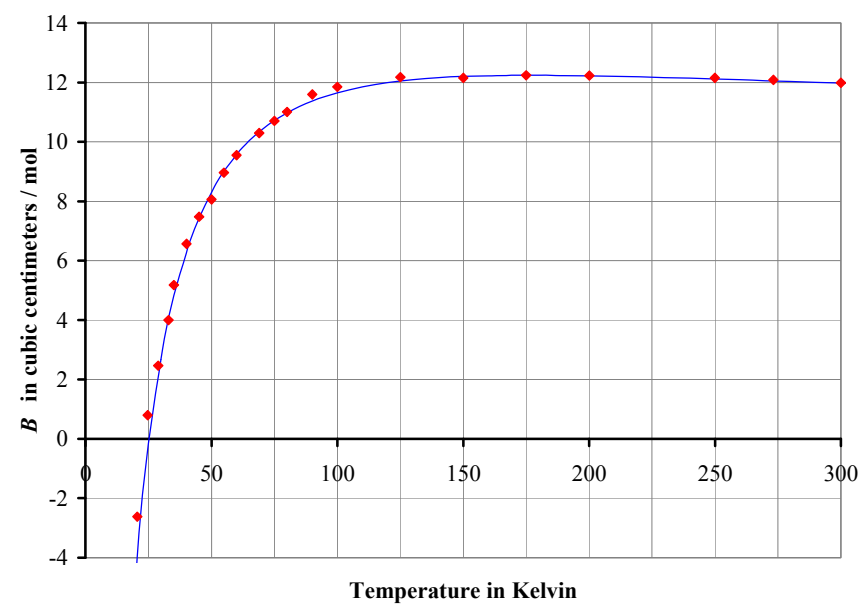

Fig. (2). Fitted curve for $B(T)$ Helium data.

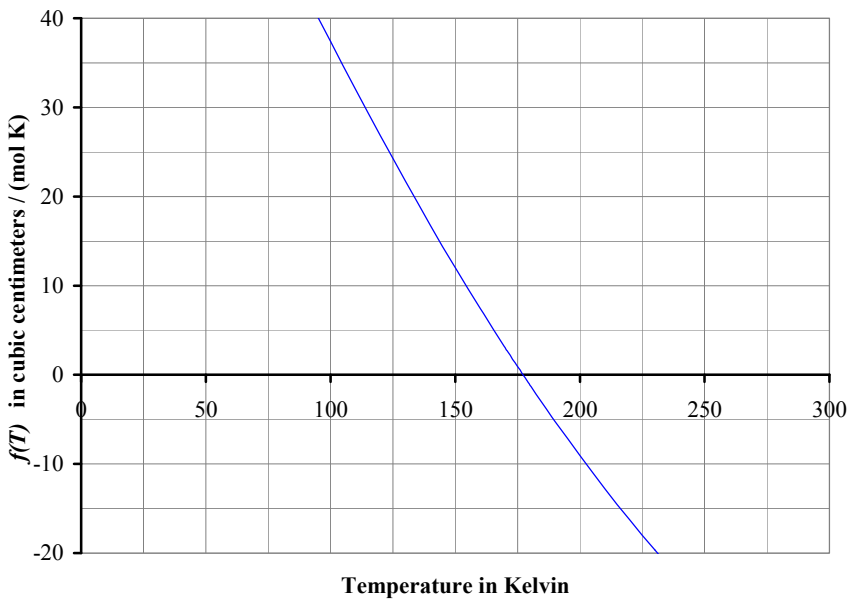

Fig. (3). $f(T)$ versus $T$ for Helium, resulting $T_{i} \approx 177 \mathrm{~K}$.

\section{$(24,12)$ LENNARD-JONES POTENTIAL}

According to Callen [1], the experimental Joule inversion temperature for hydrogen is $T_{i} \approx 400 \mathrm{~K}$. By means of a LJpotential, Goussard and Roulet [2] calculated $T_{i} \approx 800 \mathrm{~K}$. However, if one uses the algebraic expression for $B(T)$ given by Holborn and Otto $[3,5]$ the resulting $T_{i}$ is $474 \mathrm{~K}$, no so far the accepted experimental value $T_{i} \approx 400 \mathrm{~K}$. In section 3 , we 
found that by means of a LJ-potential modified by using a Jagla type ramp the Joule inversion temperature for $\mathrm{H}_{2}$ is $T_{i}$ $\approx 922 \mathrm{~K}$. However, if we use a Lennard-Jones potential with exponents $(12,6), B(T)$ can be calculated as an infinite power series $[3,12]$.

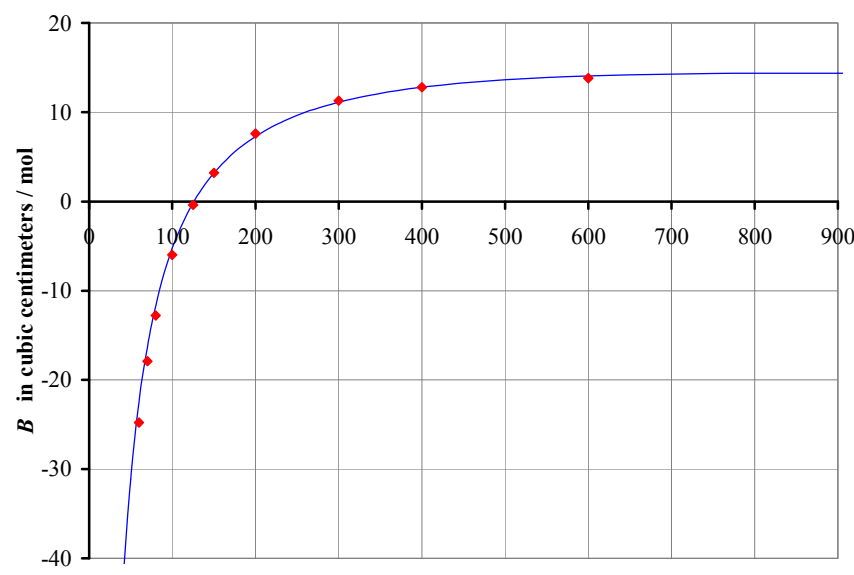

Temperature in Kelvin

Fig. (4). Fitted curve for $B(T)$ Neon data.

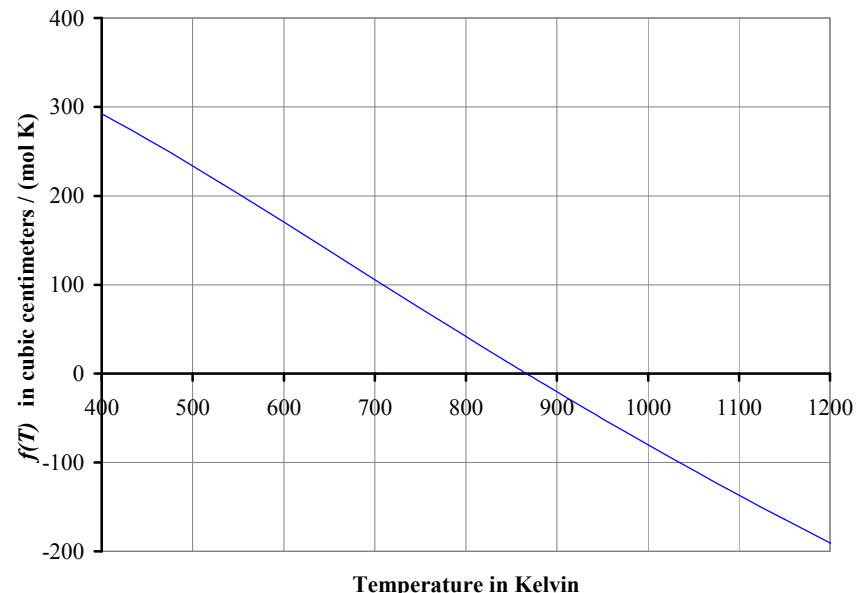

Fig. (5). $f(T)$ versus $T$ for Neon, resulting $T_{i} \approx 867 \mathrm{~K}$.

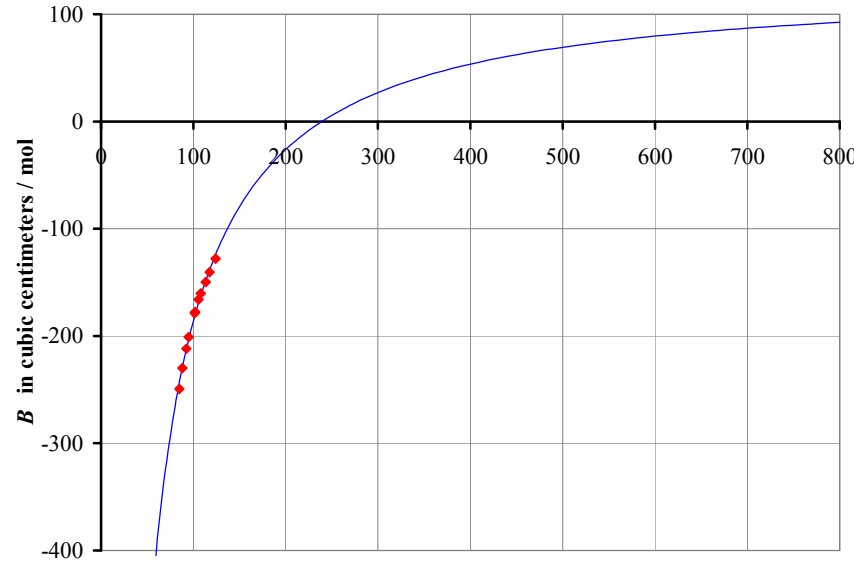

Temperature in Kelvin

Fig. (6). Fitted curve for $B(T)$ Argon data.

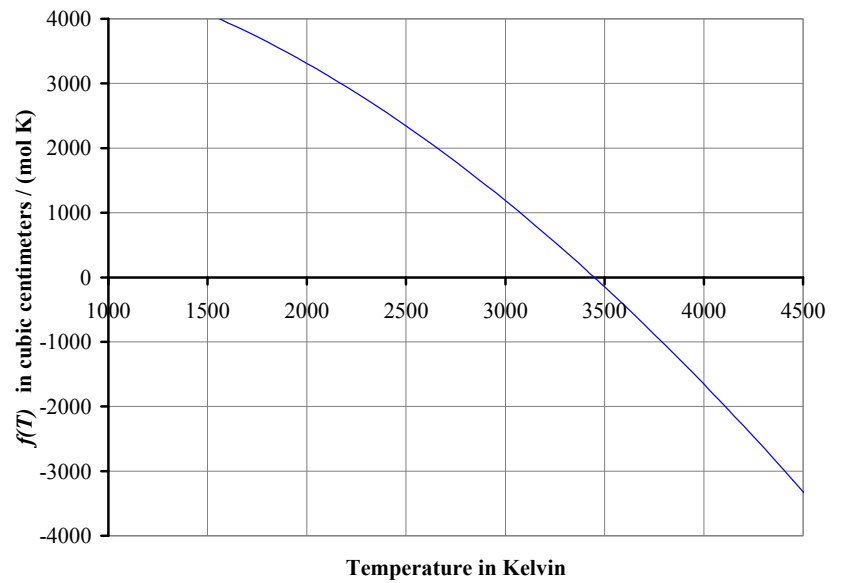

Fig. (7). $f(T)$ versus $T$ for Argon, resulting $T_{i} \approx 3448 \mathrm{~K}$.

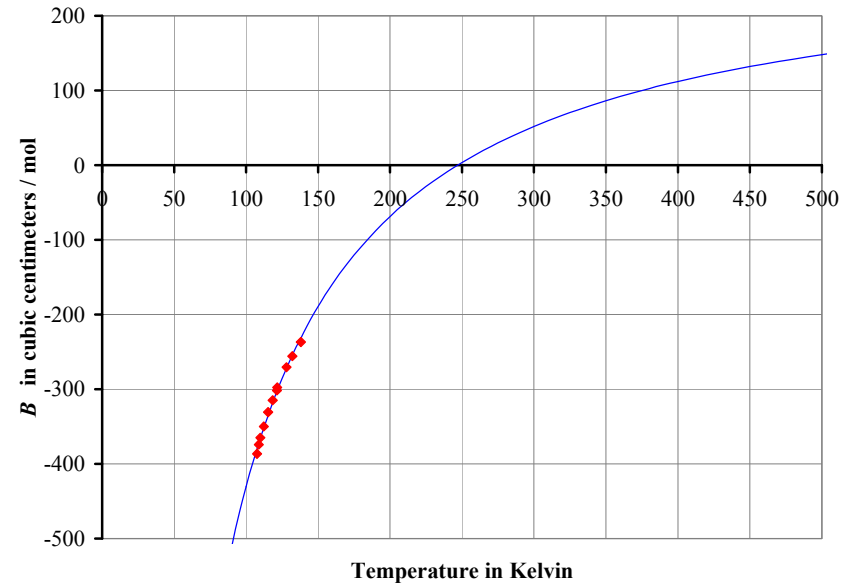

Fig. (8). Fitted curve for $B(T)$ Krypton data.

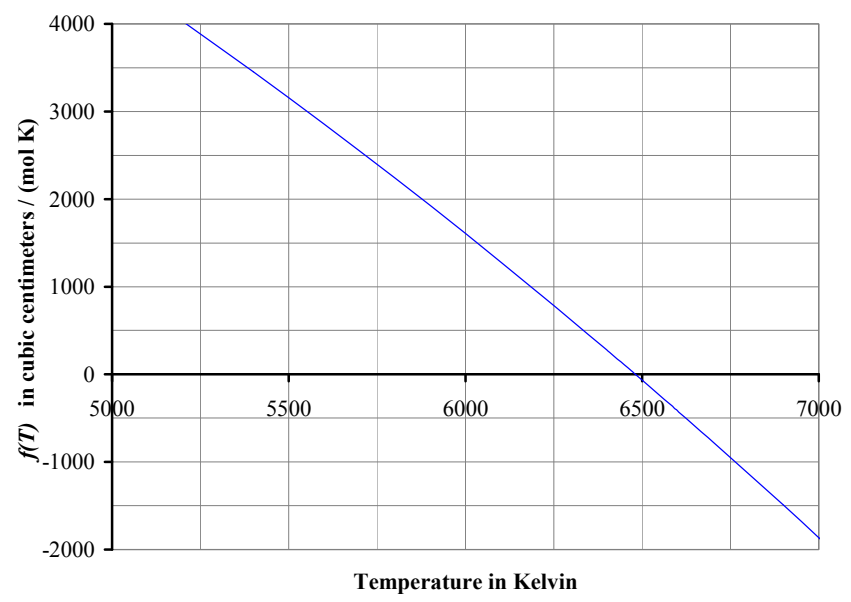

Fig. (9). $f(T)$ versus $T$ for Krypton, resulting $T_{i} \approx 6481 \mathrm{~K}$.

$$
B(T)=b_{0} \sum_{j=0}^{\infty} b^{(j)} T^{*-\left(\frac{2 j+1}{4}\right)}
$$

being $T^{*}=k T / \varepsilon$ the reduced temperature, $b_{0}=(2 / 3) \pi N_{A} \sigma^{3}$ is four times the volume of a molecule multiplied by $N_{A}$, and 


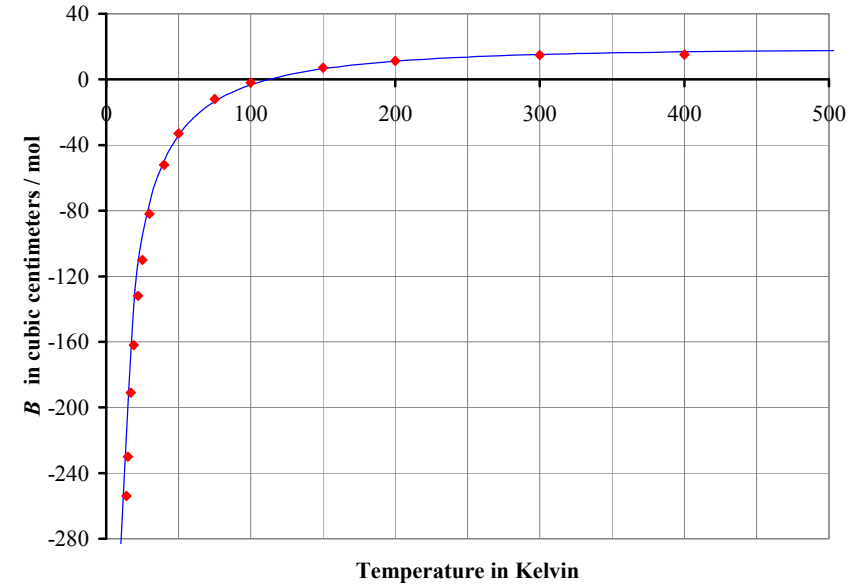

Fig. (10). Fitted curve for $B(T)$ Hydrogen data.

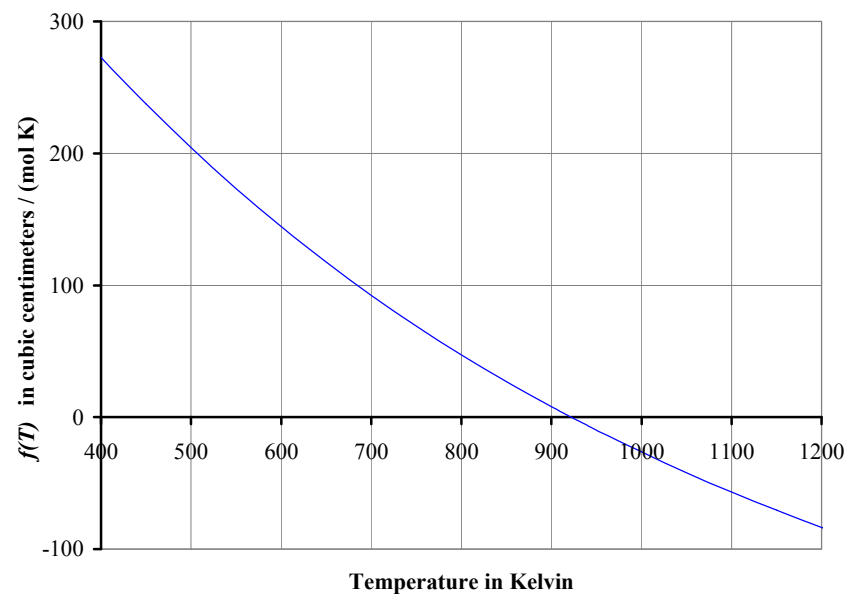

Fig. (11). $f(T)$ versus $T$ for Hydrogen, finding $T_{i} \approx 922 \mathrm{~K}$.

$b^{(j)}=-\frac{2^{j+\frac{1}{2}}}{4 j !} \Gamma\left(\frac{2 j-1}{4}\right)$

By means of $d B / d T=0$ and taking $j=5$ one finds [3]

$T^{*}=\frac{k T_{i}}{\varepsilon} \approx 25.125$

which leads to $T_{i} \approx 930 \mathrm{~K}$ for hydrogen; that is a value far from the recognized experimental value, $T_{i} \approx 400 \mathrm{~K}$. On the other hand, if we take the LJ-potential in the form [13],

$$
u(r)=4 \varepsilon\left[\left(\frac{\sigma}{r}\right)^{2 n}-\left(\frac{\sigma}{r}\right)^{n}\right]
$$

in terms of the reduced temperature $T^{*}$ for the second virial coefficient, we obtain,

$$
B^{*}\left(T^{*}, 2 n, n\right)=-\sum_{j=0}^{\infty} \frac{3}{2 j ! n} \Gamma\left(\frac{n j-3}{2 n}\right)\left(\frac{4}{T^{*}}\right)^{(n j+3) / 2 n}
$$

This expression, by means of $d B / d T=0$ gives us the reduced inversion temperatures shown in Table $\mathbf{1}$.
Table 1. $T_{i}^{*}$ As Function of LJ-Potentials $(2 n, n)$

\begin{tabular}{|c|c|}
\hline $\mathbf{L J}(\mathbf{2 n}, \boldsymbol{n})$ & $\boldsymbol{T}_{i}{ }^{*}$ \\
\hline \hline $\mathrm{LJ}(8,4)$ & 90 \\
\hline $\mathbf{L J}(\mathbf{1 2}, \mathbf{6})$ & $\mathbf{2 5 . 1 5}$ \\
\hline $\mathrm{LJ}(16,8)$ & 17 \\
\hline $\operatorname{LJ}(20,10)$ & 14 \\
\hline $\mathbf{L J}(\mathbf{2 4}, \mathbf{1 2})$ & $\mathbf{1 2 . 4 7}$ \\
\hline $\operatorname{LJ}(28,14)$ & 11 \\
\hline $\operatorname{LJ}(48,24)$ & 9.7 \\
\hline $\operatorname{LJ}(96,48)$ & 8.65 \\
\hline
\end{tabular}

It is remarkable that for the exponents $(24,12)$ which corresponds to a very short ranged potential [14], $T_{i}^{*}=$ 12.47. Thus by using $\varepsilon \approx 28 \times 10^{-4} \mathrm{eV}$ for hydrogen $[2,15]$, we obtain a Joule inversion temperature of $T_{i}\left(\mathrm{H}_{2}\right) \approx 400 \mathrm{~K}$ in agreement with the experimental value given by Callen [1]. In Fig. (12) we depict a comparison between the LJ-potential for both pairs of exponents $(12,6)$ and $(24,12)$.

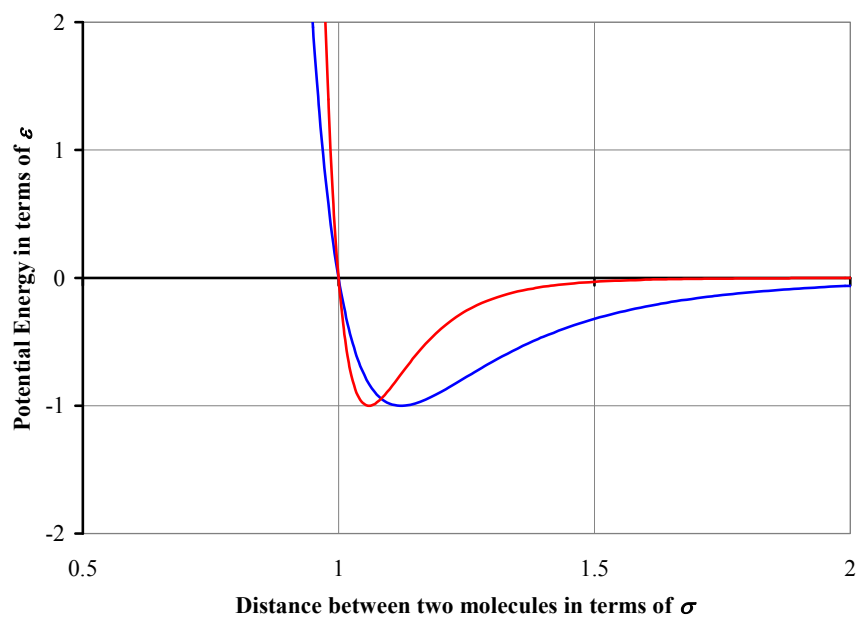

Fig. (12). LJ-potential $(12,6)$ is plotted by using a blue line. The red line shows the LJ-potential $(24,12)$, [14].

\section{CONCLUDING REMARKS}

Although the throttling Joule-Kelvin expansion is a process widely presented in thermodynamics textbooks, the so-called Joule expansion for real gases is scarcely discussed in the literature [2-4]. Goussard and Roulet [2] found that all real gases which can be described by an intermolecular potential $u(r)$ diverging for small values of $r$ at least like $r^{-3}$, necessarily have an inversion temperature. These authors calculated $T_{i}$ for $\mathrm{He}$ and $\mathrm{H}_{2}$ by using a Lennard-Jones potential finding a good result for $\mathrm{He}$ and twice to big than its estimated experimental value for $\mathrm{H}_{2}$. On the other hand, Boschi-Filho and Buthers [3] calculated $T_{i}$ for several real gases at high temperatures by using $B(T)$ behavior and they concluded that most of simple real gases have $T_{i}$ 's in a range of temperatures so high that few experimental data are available.

In the present paper, we have calculated Joule inversion temperatures for some simple real gases by means of a 
Table 2. Calculated Values for $\lambda, \sigma$ and $\varepsilon / k$ by Fitting the Experimental Data to Eq. (13). The Last Column Shows the $\varepsilon / k$ Values Provided by Poling et al. [9]

\begin{tabular}{|c|c|c|c|c|}
\hline Gas & $\lambda\left[\times \mathbf{1 0}^{-\mathbf{1 0}} \mathbf{m}\right]$ & $\boldsymbol{\sigma}\left[\times \mathbf{1 0} \mathbf{- 1 0}^{-\mathbf{m}]}\right.$ & $\varepsilon / \boldsymbol{k}[\mathbf{K}]$ & $\varepsilon / \boldsymbol{k}[\mathbf{K}] \mathbf{b y}$ Poling et al. $[\mathbf{9}]$ \\
\hline \hline $\mathbf{H e}$ & 2.002807 & 2.477172 & 9.1289 & 10.22 \\
\hline $\mathbf{N e}$ & 2.027787 & 2.571497 & 45.7158 & 32.80 \\
\hline $\mathbf{A r}$ & 3.145084 & 4.746780 & 89.1935 & 93.30 \\
\hline $\mathbf{K r}$ & 3.631624 & 6.153939 & 92.4413 & 178.90 \\
\hline $\mathbf{H}_{\mathbf{2}}$ & 2.368190 & 2.861934 & 39.9372 & 59.70 \\
\hline
\end{tabular}

Table 3. Joule Inversion Temperatures for Several Real Gases. The Second Column Shows Calculated $T_{i}$ by Using Eq. (14). Also, the Calculated $T_{i}$ 's by Goussard and Roulet [2] and Those Calculated by Boshi-Filho and Buthers [3] are Shown in Third and Fourth Columns Respectively

\begin{tabular}{|c|c|c|c|}
\hline Gas & $T_{i}[\mathrm{~K}]$ by Using eq. (14) & $T_{i}[\mathrm{~K}]$ by $[2]$ & $T_{i}[\mathbf{K}]$ by $[3]$ \\
\hline $\mathrm{He}$ & 177 & 200 & $200 \pm 50$ \\
\hline Ar & 3448 & ----- & $3800 \pm 1800$ \\
\hline $\mathbf{K r}$ & 6481 & ----- & ----- \\
\hline
\end{tabular}

Lennard-Jones potential modified by a Jagla type ramp, obtaining $T_{i}$-results within a range similar to previous reported values [2, 3]. In Table 2 we present our obtained results for $\lambda, \sigma$ and $\varepsilon / k$, according to experimental data and equation (13). In the same Table 2, we show the values for $\varepsilon / k$ provided by Poling et al. [9]. In Table 3 we show the Joule inversion temperatures obtained by using equation (14) and the results obtained by [2, 3]. However, for the case of hydrogen, we found that a Lennard-Jones potential with $(24,12)$ exponents is more suitable to calculate a $T_{i}$ near its estimated experimental value of $T_{i} \approx 400 \mathrm{~K}$.

\section{ACKNOWLEDGEMENTS}

This work was partially supported by COFFA-EDI-IPN, México.

\section{REFERENCES}

[1] H. B. Callen, Thermodynamics and an Introduction to Thermostatistics. New York: John Wiley and Sons, 1985.

[2] J. O. Goussard, and B. Roulet, "Free expansion for real gases", Am. J. Phys., vol. 61, pp. 845-848, January 1993.

[3] H. Boschi-Filho, and C. C. Buthers, "Second virial coefficient for real gases at high temperature", eprint arXiv:cond-mat/9701185 v2 28, Jan 1997. [Online]. Available: http://arxiv.org/PS_cache/ cond-mat/pdf/9701/9701185v2.pdf [Accessed March 15, 2009].

[4] G. A. Parsafar, M. Khanpour, and A. A. Mohammadi, "Calculation of equilibrium and transport properties using modified hardcore potential models", Chem. Phys., vol. 326, pp. 527-534, March 2006.
[5] L. Holborn, and J. Otto, "Über die Isothermen einiger Gase zwischen $+400^{\circ}$ und $-183^{\circ}$ ", (in German), Z. Physik, vol. 33, pp. 1-11, May 1925.

[6] L. Xu, I. Ehrenberg, S. V. Buldyrev, and H. E. Stanley, "Relationship between the liquid-liquid phase transition and dynamic behaviour in the Jagla model", J. Phys. Condens. Matter, vol. 18, pp. S2239-S2246, August 2006.

[7] J. M. Prausnitz, R. N. Lichtenthaler, and Y. E. Gomes de Azevedo, Termodinámica Molecular de los Equilibrios de Fases, (in Spanish). Madrid: Prentice Hall, 2000.

[8] D. White, T. Rubin, P. Camky, and H. L. Johnston, "The virial coefficients of helium from 20 to $300^{\circ} \mathrm{K}$ ", J. Phys. Chem., vol. 64, pp. 1607-1612, January 1960.

[9] B. E. Poling, J. M. Prausnitz, and J. P. O'Connell, The Properties of Gases and Liquids. New York: McGraw Hill, 2000.

[10] J. D. Dymond, and E. B. Smith, The Virial Coefficients of Pure Gases and Mixtures. Oxford: Clarendon Press, 1980.

[11] B. E. F. Fender, and G. D. Halsey, Jr., "Second virial coefficients of argon, krypton, and argon-krypton mixtures at low temperatures", J. Chem. Phys., vol. 36, pp. 1881-1888, October 1962.

[12] J. O. Hirschfelder, C. F. Curtiss, and R. B. Bird, Molecular Theory of Gases and Liquids. New York: Wiley, 1954.

[13] M. L. Glasser, "Second virial coefficient for a Lennard-Jones (2n-n) system in $d$ dimensions and confined to a nanotube surface", Phys. Lett. A, vol. 300, pp. 381-384, June 2002.

[14] G. A. Vliegenthart, J. F. M. Lodge, and H. N. W. Lekkerkerker, "Strong weak and metaestable liquids structural and dynamical aspects of the liquid state", Phys. A, vol. 263, pp. 378-388, February 1999.

[15] Shang-Keng Ma, Statistical Mechanics. Philadelphia: World Scientific, 1985. 